\title{
EFFECT OF INTERFACE INVERSION ON THERMAL STRESS FIELD IN CZ CRYSTAL GROWTH OF OXIDE
}

\author{
TAKAO TSUKADA AND MitsunORI HOZAWA \\ Chemical Research Institute of Non-Aqueous Solutions, \\ Tohoku University, Sendai, 980 \\ NOBUYUKI IMAISHI \\ Institute of Advanced Material Study, \\ Kyushu University, Kasuga 816
}

Key Words: Crystal Growth, Oxide, Sapphire, Czochralski Method, Interface Inversion, Thermal Stress, Finite Element Method

\begin{abstract}
For $\mathrm{CZ}$ crystal growth of an oxide $\left(\mathrm{Al}_{2} \mathrm{O}_{3}\right)$, finite element analyses of the thermal stress field in the crystal are carried out, and the effect of interface shape on the maximum shear stress in the crystal is investigated.

When the crystal rotation rate increases, with other operating conditions unchanged, the melt/crystal interface changes its shape from convex to concave toward the melt at the critical Reynolds number $\left(\operatorname{Re}_{s}^{*}\right)$. When $\boldsymbol{R e}_{s}<\boldsymbol{R} e_{s}^{*}$, the maximum value of the thermal stress on the interface is insensible to $R e_{s}$ and its radial profile is an inversed $\mathrm{W}$-shape. When $\boldsymbol{R} \boldsymbol{e}_{s}$ exceeds $R e_{s}^{*}$, however, thermal stress on the interface increases very rapidly and its radial profile becomes $W$-shaped.
\end{abstract}

\section{Introduction}

In Czochralski (CZ) crystal growth of some oxides, it is well-known that the melt/crystal interface changes from convex to concave toward the melt as the crystal rotation rate or crystal diameter increases. ${ }^{1,2)}$ This abrupt change is called 'interface shape transition' or 'interface inversion', and has been considered to be caused by the change of dominant melt flow near the interface from free convection to forced convection driven by the crystal rotation. Melt/crystal interface shape is one of the critical parameters which determine the quality of a crystal. ${ }^{5-7)}$ It is necessary to acquire correct knowledge about the factors which determine the melt/crystal interface shape and also knowledge about the thermal stress in crystals with non-flat melt/crystal interface.

Recently, we ${ }^{13)}$ carried out a finite element analysis of the temperature and flow fields in the melt and the crystal of oxides such as sapphire $\left(\mathrm{Al}_{2} \mathrm{O}_{3}\right)$, taking the shapes of melt/crystal and gas/melt interfaces into account. The results revealed that the crystal diameter and the interface shape change with crystal rotation rate, and that at a certain Reynolds number the interface inversion takes place abruptly, corresponding to a change of flow mode.

For the thermal stress field in $\mathrm{CZ}$ crystal growth, a number of theoretical studies ${ }^{4,8-11)}$ have been carried out. However, there has been no theoretical study dealing with the effect of the interface inversion

Received April 17, 1989. Correspondence concerning this article should be addressed to T. Tsukada. on thermal stress. Recently, we ${ }^{14)}$ reported the effect of a radiation shield inserted in the furnace on the thermal stress in large $\mathrm{Si}$ crystals with non-flat melt/crystal interfaces (only concave to the melt). In this work, thermal stress analysis in $\mathrm{Al}_{2} \mathrm{O}_{3}$ was carried out by use of the finite element method, and the effect of the melt/crystal interface shape on the thermal stress in the crystal, especially near the solidification front, was investigated.

\section{Theory}

Figure 1 shows the cylindrical coordinate system used in our calculation. Thermal stresses in a crystal are calculated by use of a finite element model, in which it is assumed that the material is linearly elastic and isotropic, and that the thermoelastic field is axisymmetric.

In the thermal stress analysis, the following equations, i.e. the constitutive equation, the straindisplacement equation and the equilibrium equation, and the boundary conditions are necessary. The constitutive equation for a linear elastic material is given as follows.

$$
\boldsymbol{\sigma}=\boldsymbol{D}\left(\boldsymbol{\varepsilon}-\boldsymbol{\varepsilon}_{0}\right)
$$

where $\varepsilon_{0}$ is initial strain and is related to the temperature distribution in the crystal which is obtained by a thermal-convective analysis of a $\mathrm{CZ}$ system, such as in our previous work. ${ }^{13)}$ The finite element method is applied to the thermal stress analysis by using four-noded quadrilateral elements and mesh discretization. A vector $\boldsymbol{d}$, which denotes 


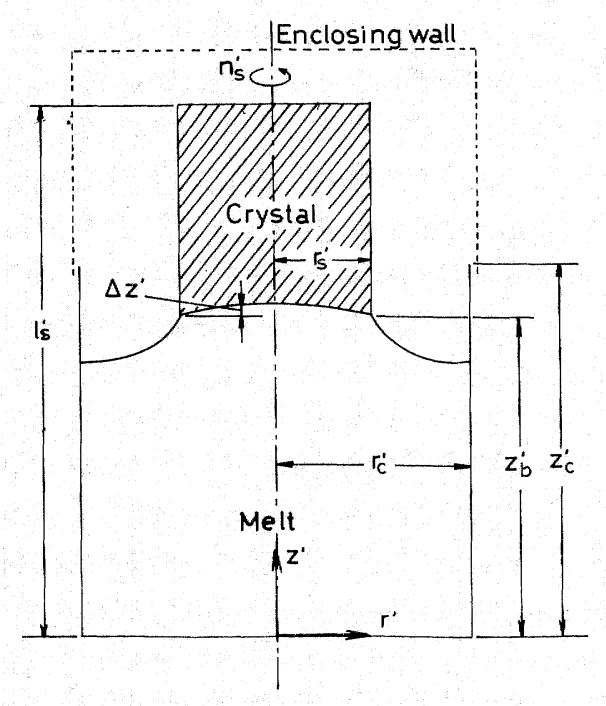

Fig. 1. Cylindrical coordinate system

the displacement field, is approximated by nodal displacement $\boldsymbol{a}$ in each element.

$$
d=\phi a
$$

With the displacements at all nodal points given by Eq. (2), the strain at any point can be described by

$$
\varepsilon=B a
$$

where $\boldsymbol{B}$ is the matrix derived from the derivatives of shape functions. With the assumptions of zero body force and surface traction free, the virtual work principle enables the equilibrium equation to be written as follows.

$$
\int_{V} \delta \varepsilon^{T} \sigma d V=0
$$

Substitution of Eqs. (1) and (3) into Eq. (4) gives the set of algebraic equations expressed by Eq. (5).

$$
\int_{V} \boldsymbol{B}^{T} \boldsymbol{D} \boldsymbol{B} d V \boldsymbol{a}=\int_{V} \boldsymbol{B}^{T} \boldsymbol{D} \varepsilon_{0} d V
$$

Accordingly, the displacement at each nodal point can be obtained by solving the above equation under the boundary condition in which radial displacement at center axis is equal to zero. Finally, the thermal stresses in the crystal can be given by Eq. (1), using the displacements obtained and Eq. (3).

In our finite element model for thermal stress analysis, the final process is to give the maximum shear stress defined by Eq. (6)

$$
\tau_{\max }=\left(\sigma_{1}-\sigma_{3}\right) / 2
$$

where $\sigma_{1}$ and $\sigma_{3}$ are principal stress $\left(\sigma_{1}>\sigma_{2}>\sigma_{3}\right)$.

\section{Results and Discussion}

\subsection{Interface shape change with crystal rotation} $\mathrm{We}^{13)}$ have computed the temperature and flow

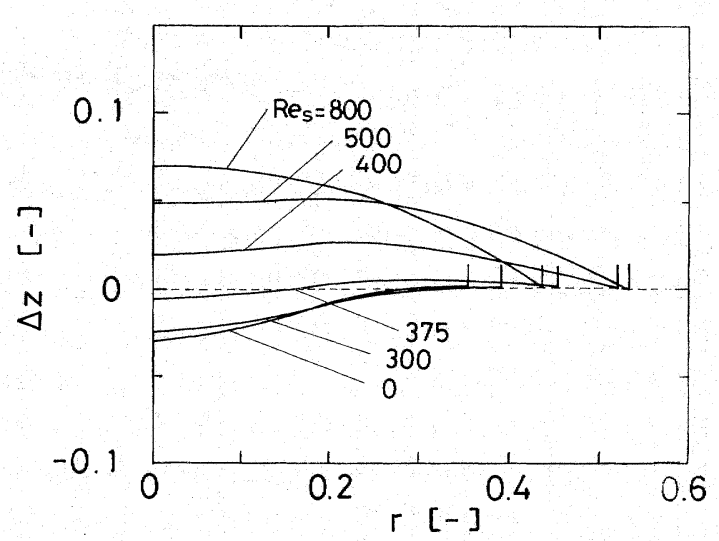

Fig. 2. Effect of crystal rotation rate on melt/crystal interface shape

Table 1. Operating conditions

\begin{tabular}{lc} 
Crucible radius $\left(r_{c}^{\prime}\right)[\mathrm{cm}]$ & 1.0 \\
Crucible height $\left(z_{c}^{\prime}\right)[\mathrm{cm}]$ & 1.9 \\
Crystal length $\left(l_{s}^{\prime}\right)[\mathrm{cm}]$ & 2.7 \\
Melt volume $\left(V_{m}^{\prime}\right)\left[\mathrm{cm}^{3}\right]$ & 4.7 \\
Enclosing wall area $\left(A_{a}^{\prime}\right)\left[\mathrm{cm}^{2}\right]$ & 10.0 \\
Enclosing wall temperature $\left(T_{a}^{\prime}\right)[\mathrm{K}]$ & 1737 \\
Crucible temperature $\left(T_{c}^{\prime}\right)[\mathrm{K}]$ & 2473 \\
Crystal rotation rate $\left(n_{s}^{\prime}\right)\left[\mathrm{min}^{-1}\right]$ & $0-68$ \\
Pull rate $\left(V_{s}^{\prime}\right)\left[\mathrm{mm} \mathrm{min}^{-1}\right]$ & 0.1 \\
\hline
\end{tabular}

fields in the melt and the crystal, and the shapes of melt/crystal and melt/gas interfaces for $\mathrm{Al}_{2} \mathrm{O}_{3}$. Figure 2 shows the effect of crystal rotation rate $\left(R e_{s}\right)$ on the shape of the melt/crystal interface under the operating conditions shown in Table 1, where $\Delta z$ is axial displacement of the interface from a plane. It is found that the flow field strongly influences the interface shape, and the interface inversion from convex to concave toward melt occurs approximately at $R e_{s}=375$. This value is in good agreement with the critical Reynolds number $R e_{s}^{*}(=379)$ predicted by Kobayashi's correlation, ${ }^{3)}$ combined with the radius of the crystal shown in Fig. 2.

\subsection{Thermal stress distribution}

The effect of the melt/crystal interface shape on the thermal stress field in $\mathrm{Al}_{2} \mathrm{O}_{3}$ crystal is discussed below by use of the following Young's modulus $E^{15)}$ and linear expansion coefficient $\beta^{12)}$.

$$
\begin{aligned}
E= & -60.94 \times\left(T^{\prime}-273.15\right)+3.62 \times 10^{5} \\
\beta= & 5.927 \times 10^{-6}+4.284 \times 10^{-9} T^{\prime} \\
& -6.621 \times 10^{-13} T^{\prime 2} \quad\left[\mathrm{~K}^{-1}\right]
\end{aligned}
$$

Poisson's ratio $v$ is assumed to be constant, $v=0.25$, because of the lack of reliable data.

Figure 3 shows typical results of the isotherms (on the left) and the isostress contours of maximum shear stresses, $\tau_{\max }$, defined by Eq. (6) (on the right), at $R e_{s}=0,375$ and 800 , respectively. In crystals with a flat or convex interface (Fig. $3 a$ and $b$ ), $\tau_{\max }$ on the 


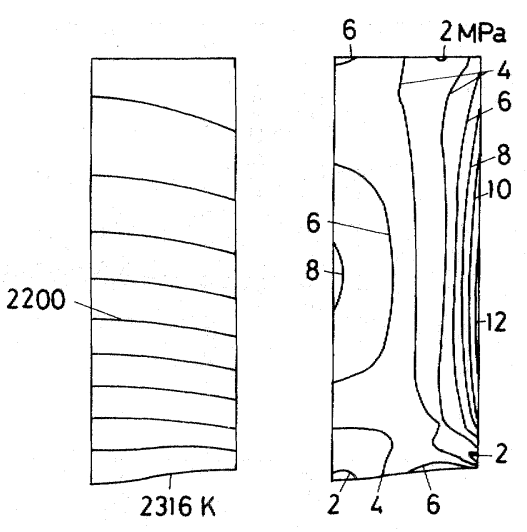

(a) $\operatorname{Re}_{\mathrm{s}}=0$

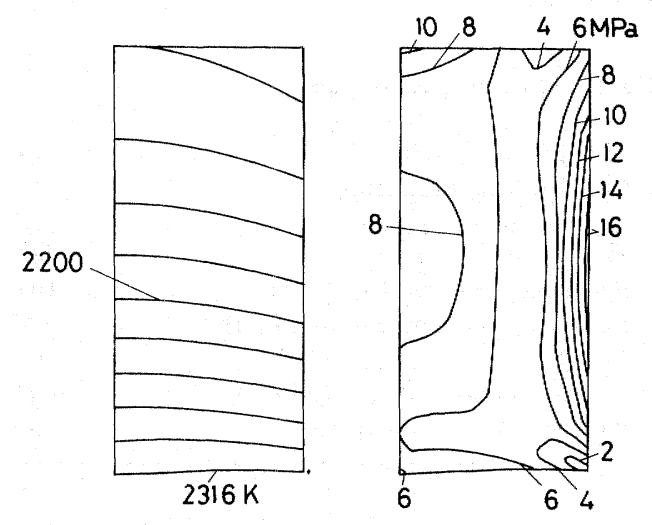

(b) $R e_{S}=375$

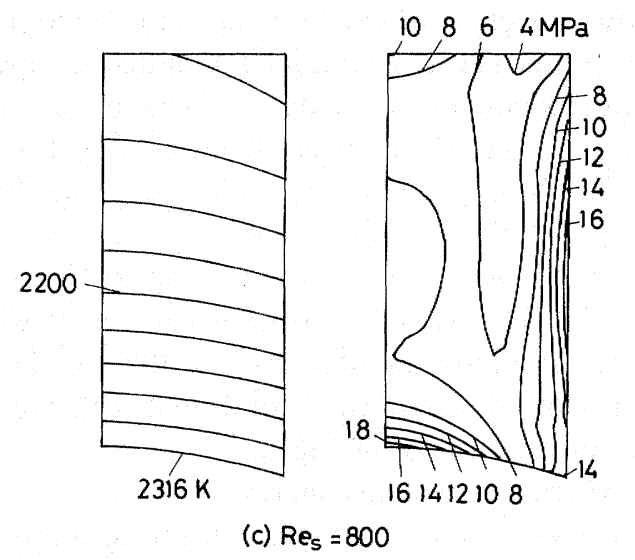

Fig. 3. Isotherms and isostress contours of maximum shear stress

melt/crystal interface is small, and the maximum value of $\tau_{\max }$ appears on the side wall of the crystal. In the crystal with a concave interface (c), large $\tau_{\max }$ appears on the melt/crystal interface, especially near the center axis, as well as on the crystal side wall.

Figure 4 shows the radial distributions of $\tau_{\max }$ on the melt/crystal interface at different $R e_{s}$. For the convex interfaces $\left(R e_{s}=0-350\right), \tau_{\max }$ on the melt/ crystal interface shows a maximum at a certain radius $r=r^{+}$, and $r^{+}$decreases as $R e_{s}$ increases, while for a concave interface $\left(R e_{s}>R e_{s}^{*}\right), \tau_{\max }$ on the melt/crystal interface shows a local minimum at radius $r=r^{-}$. On

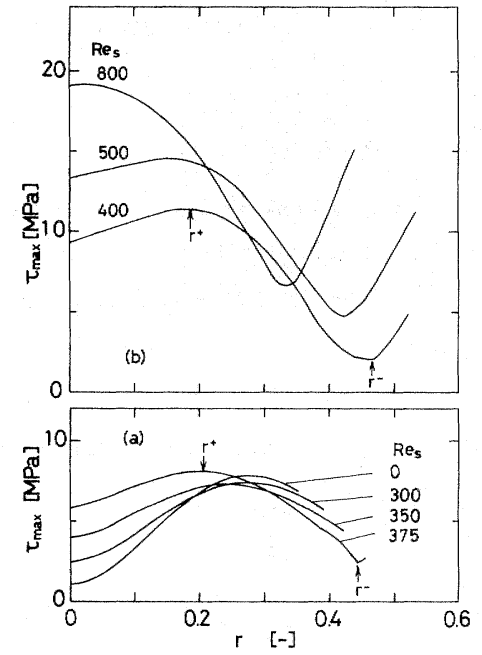

Fig. 4. Distribution of "maximum shear stress on melt/ crystal interface at various Reynolds numbers

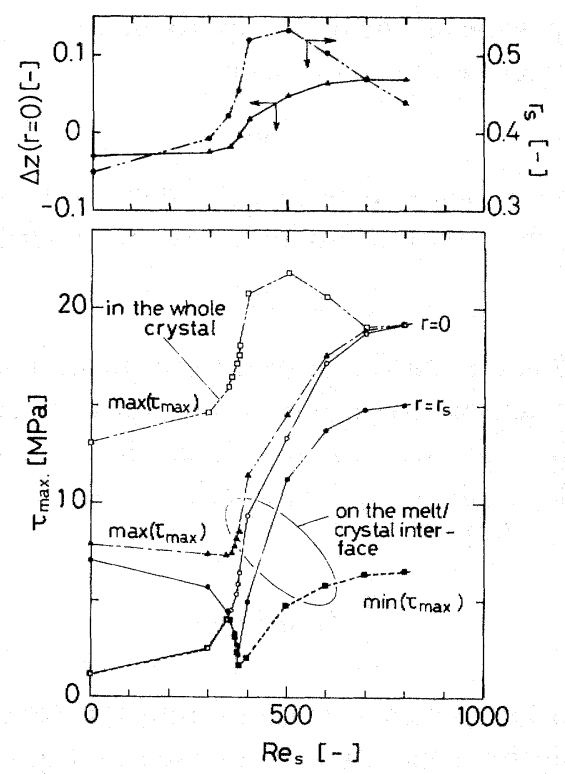

Fig. 5. Effect of crystal rotation rate on maximum shear stress at various points in the crystal, and on melt/crystal interface shape and crystal radius

the other hand, $r^{+}$to give a maximum for $\tau_{\max }$ continues to decrease as $R e_{s}$ increases and at $R e_{s}=800, r^{+}$ becomes zero.

Figure 5 shows the effect of crystal rotation rate, $R e_{s}$, on the maximum shear stress $\tau_{\max }$ at various points in the crystal. Fig. 5 also shows the changes of axial displacement of the interface from a flat plane at $r=0$, $\Delta z$, and the crystal radius, $r_{s}$, with $R e_{s} . \tau_{\max }$ at $r=r_{s}$ shows a minimum value at $R e_{s}=380$ where the interface shape is almost flat, while, $\tau_{\max }$ at $r=0$ shows the minimum at $R e_{s}=0$ and increases with $R e_{s}$, exclusively steeply near the $R e_{s}^{*}$. The minimum of $\tau_{\max }$ on the interface appears at $r=0$ when $R e_{s}$ is small, and then moves outward to the periphery of the crystal as $R e_{s}$ increases beyond $R e_{s}^{*}$. After the interface 
inversion occurs at $R e_{s}=375$, the minimum value of $\tau_{\max }$ is observed at $r^{-}$. These results can be explained as follows. When the melt/crystal interface is non-flat, there always exists a radial temperature gradient near the periphery of the crystal bottom $\left(r=r_{s}, z=z_{b}\right)$ and it becomes larger as the non-flatness of the interface increases. Accordinly, $\tau_{\max }$ at $\left(r_{s}, z_{b}\right)$ becomes large at both $R e_{s}=0$ (convex interface to the melt) and 800 (concave interface), due to the large radial temperature gradients. At the center axis the axial temperature gradient and the crystal radius increase with $R e_{s}$, and thus $\tau_{\max }$ at $r=0$ increases with $R e_{s}$.

Figure 5 also shows the effect of $R e_{s}$ on the maximum values of $\tau_{\max }$ both on the melt/crystal interface and in the whole crystal. From Fig. 5, the maximum value of $\tau_{\max }$ on the interface is almost constant at $R e_{s}<R e_{s}^{*}$ and then increases rapidly after the interface inversion occurs. $\max \left(\tau_{\max }\right)$ in a whole crystal also increases rapidly after the interface inversion and then decreases. The decrease of $\max \left(\tau_{\max }\right)$ corresponds to the decrease of crystal radius in this region. Also, for large $R e_{s}$, the maximum value of $\tau_{\max }$ in a whole crystal coincided with that on the melt/crystal interface.

In the present results, the maximum of $\tau_{\max }$ in a whole crystal appears on the side wall of a crystal, which seems quite unrealistic. This comes from the thermal boundary condition of a uniform ambient wall temperature, posited on our previous work, for the sake of simplification. In a growing crystal in a practical CZ furnace, the maximum value of $\tau_{\max }$ may appear near the top of the crystal rod, where the crystal surface is faced to the coldest ambient wall and radiative heat loss is exclusively large. Accordingly, at the upper part of the crystal, more heat flows axially upward than predicted by the present thermal model. But the present models reasonably represent the temperature and stress fields near the melt crystal interface. These results first revealed that the stress-free melt/crystal interface is practically unrealistic. A non-flat melt/crystal interface is always thermally stressed and the stress distribution on it varies with the operating conditions, such as $R e_{s}$.

The present work is limited to a qualitative study, because there were no experimental data for comparison. To achieve the design of a $\mathrm{CZ}$ puller by such a computer simulation as we propose, we have to know a limited highest shear stress value to produce high-quality crystal, which requires accumulated experimental information about the relationship between crystals quality and the operating conditions.

\section{Conclusions}

For $\mathrm{CZ}$ crystal growth of $\mathrm{Al}_{2} \mathrm{O}_{3}$, finite element analyses of thermal stress in the crystal were carried out to investigate the effect of interface inversion on the thermal stress distribution. In a crystal with a non-flat melt/crystal interface, the interface is always exposed to the thermal stress.

The thermal stress field in the crystal is affected strongly by the shape of the melt/crystal interface, and the maximum shear stress on the melt/crystal interface increases rapidly at $R e_{s}$ values larger than $R e_{s}^{*}$, i.e. after the interface inversion from convex to the melt toward concave occurs.

\section{Nomenclature}

$A_{a}^{\prime} \quad=$ enclosing wall area $\left[\mathrm{m}^{2}\right]$

$\boldsymbol{a}=$ nodal displacement vector $[\mathrm{m}]$

B $\quad=$ strain-displacement matrix $\quad[1 / \mathrm{m}]$

$\boldsymbol{D} \quad=$ elastic stress-strain matrix [Pa]

$\begin{array}{lll}\boldsymbol{d} & =\text { displacement vector } & {[\mathrm{m}]}\end{array}$

$E \quad=$ Young's modulus $\quad[\mathrm{Pa}]$

$l_{s}^{\prime} \quad=$ crystal length $\quad[\mathrm{m}]$

$n_{s}^{\prime} \quad=$ crystal rotation rate $\quad[1 / \mathrm{s}]$

$R e_{s} \quad=$ Reynolds number $\left(=\rho_{l} r_{c}^{\prime 2} n_{s}^{\prime} / \mu_{l}\right)$, [-]

$R e_{s}^{*} \quad=$ critical Reynolds number [-]

$r^{\prime} \quad=$ radial distance in cylindrical coordinates [m]

$r_{c}^{\prime} \quad=$ crucible radius $\quad$ [m]

$r_{s}^{\prime} \quad=$ crystal radius $\quad[\mathrm{m}]$

$r, r_{\mathrm{s}} \quad=r^{\prime} / r_{\mathrm{c}}^{\prime}, r_{\mathrm{s}}^{\prime} / r_{\mathrm{c}}^{\prime} \quad[-]$

$r^{+}=$radial distance at which $\tau_{\max }$ on the melt/crystal interface shows maximum value [-]

$=$ radial distance at which $\tau_{\max }$ on the melt/crystal interface shows minimum value [-]

$=$ temperature

$=$ enclosing wall temperature

$=$ crucible temperature

$=$ melt volume

$=$ pull rate

$=$ axial distance in cylindrical coordinates

$=z^{\prime} / r_{c}^{\prime}$

$=$ crucible height

$\Delta z^{\prime} \quad=$ axial displacement of melt $/$ crystal interface from a plane

$\beta \quad=$ linear thermal expansion coefficient

$\boldsymbol{\varepsilon} \quad=$ strain

$\varepsilon_{0} \quad=$ initial strain

$v \quad=$ Poisson's ratio

$\mu_{l} \quad=$ viscosity

$\rho_{s} \quad=$ density of crystal

$\sigma \quad=$ stress

$[1 / \mathrm{K}]$

$[-]$

$[-]$

$[-]$

$[\mathrm{Pa} \cdot \mathrm{s}]$

$[\mathrm{Pa}]$

$\sigma_{1}, \sigma_{2}, \sigma_{3}=$ principal stress $\quad[\mathrm{Pa}]$

$\tau_{\max } \quad=$ maximum shear stress $\quad[\mathrm{Pa}]$

$\phi \quad=$ shape function $\quad[-]$

\section{Literature Cited}

1) Cockayne, B., M. Chesswas and D. B. Gasson: J. Mater. Sci., 3, 224 (1968).

2) Cockayne, B., M. Chesswas, P. J. Born and J. D. Filby: $J$. Mater. Sci., 4, 236 (1969)

3) Kobayashi, N.: J. Crystal Growth, 54, 414 (1981).

4) Kobayashi, N. and T. Iwaki: J. Crystal Growth, 73, 96(1985).

5) Miyazawa, S. and H. Iwasaki: Japan. J. Appl. Phys., 9, 441 (1970).

6) Miyazawa, S. and S. Kondo: Mater. Res. Bull., 8, 1215 (1973).

7) Miyazawa, S.: J. Crystal Growth, 49, 515 (1980).

8) Motakef, S. and A. F. Witt: J. Crystal Growth, 80, 37 (1987) 
9) Motakef, S.: J. Crystal Growth, 88, 341 (1988).

10) Schvezov, C., I. V. Samarasekera and F. Weinberg: J. Crystal Growth, 84, 219 (1987).

11) Schvezov, C., I. V. Samarasekera and F. Weinberg: J. Crystal Growth, 85, 142 (1987).

12) Touloukian, Y. S., R. K. Kirby, R. E. Taylor and T. Y. R. Lee: Thermophysical Properties of Matter, vol 13, IFI/PLENUM, New York, 1977.
13) Tsukada, T., N. Imaishi and M. Hozawa: J. Chem. Eng. Japan, 21, 184 (1988).

14) Tsukada, T., N. Imaishi and M. Hozawa: J. Chem. Eng. Japan., 23, 186 (1990).

15) Wachtman, J. B. and D. G. Lam: J. Am. Ceram. Soc., 42, 254 (1959). 